\title{
Circuit
}

Musiques contemporaines

\section{Noli me tangere. Violence politique, éthique protestante et composition musicale dans une section de La Petite Fille aux allumettes de Helmut Lachenmann \\ Noli me tangere. Political Violence, Protestant Ethic and \\ Musical Composition in a Section of Helmut Lachenmann's The Little Match Girl}

\section{Laurent Feneyrou}

Volume 28, numéro 3, 2018

Engagements sonores : éthique et politique

URI : https://id.erudit.org/iderudit/1055191ar

DOI : https://doi.org/10.7202/1055191ar

Aller au sommaire du numéro

Éditeur(s)

Circuit, musiques contemporaines

ISSN

1183-1693 (imprimé)

1488-9692 (numérique)

Découvrir la revue

Citer cet article

Feneyrou, L. (2018). Noli me tangere. Violence politique, éthique protestante et composition musicale dans une section de La Petite Fille aux allumettes de Helmut Lachenmann. Circuit, 28(3), 13-24. https://doi.org/10.7202/1055191ar
Résumé de l'article

À partir des sections 15a et 15b de La Petite Fille aux allumettes de Helmut Lachenmann, qui mettent en musique un extrait d'une lettre de Gudrun Ensslin, amie de jeunesse du compositeur et militante dans les mouvements étudiants ouest-allemands des années 1960, puis dans les actions de la Fraction Armée Rouge, de 1970 à sa mort en prison, en 1977, cet article interroge les relations entre écriture musicale, éthique protestante et engagement politique. Décrivant la musique des deux sections et ses principes, où prime l'exigence de l'action plutôt que l'intention, il s'interroge sur un contexte biographique et sur les conditions culturelles, historiques et politiques qui rendent possible son intégration dans une oeuvre d'art à la fin du $\mathrm{xx}^{\mathrm{e}}$ siècle. 


\section{Noli me tangere}

\section{Violence politique, éthique protestante et composition musicale dans une section de La Petite Fille aux allumettes de Helmut Lachenmann}

Laurent Feneyrou

Le 13 octobre 1977, un Boeing de la Lufthansa assurant la liaison entre les Baléares et Francfort est détourné par le Commando Martyr-Halimeh, qui réclame, dans un ultimatum au chancelier Helmut Schmidt, la libération de onze prisonniers de la Fraction armée rouge (Rote Armee Fraktion, RAF) et de deux militants palestiniens détenus en Turquie. À Mogadiscio, quatre jours plus tard, dans la nuit du 17 au 18 octobre, après un périple de l'avion entre Rome, Larnaca, Bahreïn, Dubaï et Aden où le pilote Jürgen Schumann est froidement abattu, des membres de l'unité spéciale allemande Grenzschutzgruppe 9 (GSG 9) prennent d'assaut l'appareil et libèrent les otages. Le lendemain matin, dans leurs cellules de la prison de Stammheim, non loin de Stuttgart, où ils purgeaient une peine de prison à vie prononcée le 28 avril 1977, les membres de la RAF Andreas Baader et Jan-Carl Raspe sont retrouvés mortellement blessés par balles, et Gudrun Ensslin pendue.

Le 26 janvier 1997, à Hambourg, est créée La Petite Fille aux allumettes (Das Mädchen mit den Schwefelhölzern, 1990-1996), «musique avec images » de Helmut Lachenmann, dans une mise en scène d'Achim Freyer et sous la direction de Lothar Zagrosek ${ }^{1}$. Le conte de Hans Christian Andersen y est confié à deux sopranos, à quatre groupes de solistes vocaux (SATB) et à l'orchestre, sans le moindre dialogue, traduisant ainsi la solitude de l'enfant, sa marginalité et l'indifférence du monde à son sort tragique. Une théodicée de la souffrance. Dès lors, ce que l'œuvre représente, ce sont les sensations et les sentiments de la petite fille, ainsi que sa conscience. Qu'elle soit visible sur scène ou non devient indifférent. La dislocation du texte entre les voix et les instruments crée une dramaturgie où nous assistons moins à une narration qu'au déploiement de son intériorité. Non une chanteuse incarnant la petite
1. [ndlr] Cet article prolonge, en insistant sur l'éthique protestante qui traverse l'œuvre de Helmut Lachenmann, les réflexions de Feneyrou, 2017. 
2. Lachenmann, 2009 ( Des paradis éphémères" [1993]), p. 200.

3. Cité in Kaltenecker, 2001, p. 244.
4. Schut (dir.), 1987, p. 18. Voir, pour les essais, communiqués et revendications de la RAF, Rote Armee Fraktion: Texte und Materialien zur Geschichte der RAF, Berlin, ID-Verlag, 1997. fille et chantant: «J'ai froid», mais la sensation globale, à l'écoute, du froid éprouvé.

Quelle relation, a priori improbable, entre ces deux événements?

Dans le conte d'Andersen, où il perçoit une "protestation "régressive" ", Lachenmann a introduit deux autres textes: un fragment du Codex Arundel de Léonard de Vinci sur l'inquiétude de celui qui cherche la connaissance, mais prend peur devant une grotte sombre malgré le désir d'y entrer (section 18); et, nous y voilà, un extrait d'une lettre de Gudrun Ensslin, sur le «rebelle qui se met lui-même à l'écart, qui désespère face à une société de plus en plus rigide et agit de manière violente, en sortant de la légalitée » (sections 15a et b). Ou l'attentat comme déclinaison de la transgression des allumettes allumées pour se réchauffer - une chaleur que les timbres et l'harmonie, plus consonante, expriment dans quatre sections intitulées «Ritsch» (sections 12, 14, 16a et 20a) -, et la figure historique d'Ensslin comme « double noir », contrariant l'identification à l'héroïne.

Dès 1973, les prisonniers de la RAF avaient mis en place un système de communication, le «système info », dont l'objectif était de préserver la cohésion du groupe, en conservant son «identité politique» et sa «conscience révolutionnaire ». Sur décision de Baader et d'Ensslin, et par l'intermédiaire d'avocats, des messages étaient transmis aux uns et aux autres. En 1987, Lachenmann lit une anthologie de ces messages, Das Info, d'où il tire l'extrait de la lettre d'Ensslin : à la cruauté de la nature glaçante et à la société repue (table dressée pour Noël, avec son oie rôtie, et jouets derrière la vitrine d'un grand magasin) et insensible à la misère, répond non plus l'abandon à la mort, mais la résistance de la militante:

Le criminel, le fou, le suicidaire - ils incarnent cette contradiction. Ils crèvent en elle. Leur mort symbolise l'absence de perspective et l'impuissance de l'homme dans le système: soit tu te détruis, soit tu détruis les autres. Soit mort, soit égoïste. Leur mort ne montre pas seulement la perfection du système: ils ne sont pas assez criminels, ils ne sont pas assez fous, ils ne sont pas assez meurtriers, et cela implique leur mort plus rapide par le système dans le système. Leur mort montre en même temps la négation du système: leur criminalité, leur folie, leur mort est l'expression de la rébellion des sujets écrasés contre leur écrasement, non pas choses, mais êtres humains.

Et pour finir : «Écrivez sur notre peau ${ }^{4} »$.

De cette lettre, Lachenmann écrit:

Dans sa cellule, à la prison de Stammheim, Gudrun Ensslin a écrit une lettre dont la langue est parfois très laide et très agressive, mais dont le dernier paragraphe est d'une beauté poignante - il est beau parce qu'il nomme avec précision -, si bien 
que je n’y perçois pas simplement l'acceptation déchaînée de la violence et une âme détruite, mais également un amour pour les individus brisés dans l'affrontement avec la sociétés.

Dans la section 15a de La Petite Fille aux allumettes, la musique est d'autant plus menaçante que le son est étouffé et que les groupes de solistes chuchotent presque le paragraphe d'Ensslin, distribué entre les voix à partir d'une ligne rythmique commune. Le texte est, pour l'essentiel, donné aux sopranos et aux altos, les ténors et les basses, d'abord purement phonétiques, ne l'énonçant qu'ensuite. À l'exception de pizzicatos avec l'ongle et d'un saisissant accord des violoncelles et des contrebasses (mesure 245, sur Wahnsinn, «folie»), les cordes, divisées et en harmoniques, avec mouvement de l'archet du chevalet à la touche, produisent un son éthéré. Quant aux autres instruments - cuivres, pianos aux cordes graves grattés avec l'ongle ou avec des plectres, et percussions, aux peaux frottées avec une brosse et un balai, quand les baguettes ne frottent pas l'une sur l'autre -, ils offrent un exemple d'orchestration que Lachenmann dit «consonantique», imitant voyelles, consonnes ou syllabes du texte, et redoublant ainsi le rythme des voix chorales. Les six bandes magnétiques (avec des bruits, des paroles et de la musique), de plus en plus présentes, obéissent au même principe, leur diffusion, écrite en notation musicale classique, se faisant sous forme tantôt de points aux valeurs courtes, tantôt d'enveloppes, avec sforzato, laissant alors entendre un son, un mot ou une bribe de rengaine. En somme, la ligne rythmique et les groupes vocaux dirigent l'ensemble de l'effectif qui n'en est que la diffraction, et les quelques gestes échappant a priori à cette logique tentent de trouver un équivalent acoustique au texte.

\section{I}

Commentons plus avant cette section de La Petite Fille aux allumettes. Il convient de rappeler son contexte biographique. Lachenmann s'est exprimé tardivement sur Gudrun Ensslin, qu'il a connue dans sa jeunesse et dont le père, le pasteur Helmut Ensslin, appartenait à la même communauté de Tuttlingen que son père, Ernst Lachenmann, son supérieur. Cette donnée aviva une sensibilité du compositeur à la coresponsabilité de la société ouestallemande dans la violence politique, mais sans excuses pour les actions de sa cadette.

C'était une élève extrêmement douée, aux conceptions idéalistes, et dont l'humanisme enthousiaste fut peu à peu détruit par les événements politiques de cette époque-là - la remilitarisation de l'Allemagne, les ingérences des États-Unis dans le Tiers-Monde, la guerre d’Algérie, la guerre au Vietnam... Son énergie
5. Lachenmann, 2009 ("Les sons représentent des événements naturels" [2001]), p. 249. 
intellectuelle et idéaliste changea ainsi radicalement de direction, pour se muer en une incroyable amertume, une haine du système politique qui alla jusqu'à l'acceptation criminelle de la violence ${ }^{6}$.

7. En 1987, Lachenmann offre à Luigi Nono un livre de Pieter Bakker Schutt, Stammheim: Der Prozess gegen die Rote Armee Fraktion (Kiel, Neuer Malik, 1986), avec l'envoi : "Ta musique celle d'hier et celle de maintenant - appartient au monde secret (Hölderlin-Diotima-Nono), qui est le monde réel comme ce voile spectral que Gudrun Ensslin et d'autres ont essayé de déchirer. Ils se sont déchirés eux-mêmes. Sans la perspective que tu m'as montrée dans ton art, je serais aussi paralysé qu'elle. Je pense (presque tous les jours) à elle, et continue à travailler avec l'aide de ma communication télépathique avec toi " (Fondazione Archivio Luigi Nono).

Outre les souvenirs d'enfance, outre l'incendie de deux grands magasins à Francfort - première action à laquelle Ensslin participa dans la nuit du 2 au 3 avril 1968, une bravade encore anarchiste et conçue comme un fanal contre la société de consommation et la guerre du Vietnam -, outre le «voile spectral » qu'elle aurait essayé de déchirer jusqu'à s'y sacrifier? ${ }^{7}$, outre la mort obscure à Stammheim, suicide ou meurtre d'État, c'est le thème du protestantisme qui domine le discours de Lachenmann sur Ensslin et qui rend explicite une éthique transcendantale de la lutte. S'il condamne l'idéologue gauchiste des années 1960 comme nouveau visage du prêtre, intermédiaire inopérant, Lachenmann ne s'est guère exprimé sur son propre christianisme. Et s'il met en musique dans Consolation II (1968), pour 16 voix, la désolation du paysage de la Prière de Wessobrunn, où rien n'est, ni la terre, ni le ciel, ni l'arbre, ni la montagne, ni le soleil, ni la lumière, ni la lune, ni la mer, sinon Dieu tout-puissant, il ne concède que du bout des lèvres la dimension religieuse de cette œuvre.

Dans ce contexte, rappelons que Helmut Ensslin, pasteur de l'Église évangélique d'Allemagne, pétri de culture et de traditions souabes, dont le piétisme se caractérise par la tolérance et une perspective critique, lecteur de Hegel et du théologien Karl Barth, avait été partisan de l’Église confessante (Bekennende Kirche), dont faisait notamment partie Dietrich Bonhoeffer, mort en déportation pour s'être opposé au nazisme et à une Église protestante du Reich. Helmut Ensslin, qui avait lui-même été dénoncé, condamné et amnistié sous Hitler, établit ici le lien entre éthique protestante et résistance politique. En 1968, lors du procès de sa fille pour l'incendie des magasins de Francfort, il évoquera devant les caméras de télévision la personnalité de celle-ci, au style de vie certes incompréhensible pour sa génération, mais la jugeant néanmoins «animée d'une authentique ferveur» - Gudrun, quatrième des sept enfants de la famille, avait d'ailleurs séjourné aux États-Unis en 1958 dans une communauté méthodiste. Hostile lui aussi à la remilitarisation de l'Allemagne de l'Ouest, il condamnera l'incendie, ajoutant:

Une génération qui a vécu au nom du peuple et contre son propre peuple la construction des camps d'extermination, la haine contre les juifs, les exécutions de masse, ne doit pas admettre de restauration. Elle ne doit pas permettre que les espérances en un nouveau commencement, en la réforme, en une renaissance, soient perdues ${ }^{8}$. 
Et à propos de l'incendie, il parla, empreint de protestantisme, d'« euphorie de réalisation de soi, une réalisation de soi sacrée [einer ganz heiligen Selbstverwirklichung $]^{9}$ ».

Ce n'est pas le seul exemple d'un lien entre foi et engagement dans les rangs de la $\mathrm{RAF}^{10}$ : historien de l'art, Werner Meinhof, le père d'Ulrike Meinhof, autre membre historique de la première génération du groupe, était issu d'une lignée de théologiens proches des pasteurs rénitents de Hesse (Hessische Renitenz), une communauté qui avait refusé, à l'époque de Bismarck, l'intervention de l'État dans les questions ecclésiastiques - position garante de la tolérance constitutive du protestantisme allemand -, et qui défendit, sous Hitler, la liberté religieuse contre les tentatives du Reich d'encadrer les activités paroissiales. Libéral et matérialiste, Werner Meinhof considérait que le chrétien doit s'engager dans la vie concrète. Participation au culte, musique de la maison (Hausmusik) et bénédicité rythmaient la vie dans son foyer. Étudiante en psychologie et en pédagogie, sa fille pratiquera encore cette prière, la religion dictant ses combats contre la bombe atomique: «Nous ne voulons pas avoir à nous confesser une nouvelle fois devant Dieu et les hommes pour un crime contre l'Humanitét ${ }^{11}$.»

Au cours des années 1960, comme le rappelle Jörg Herrman ${ }^{12}$, l'exigence protestante et gauchiste d'une cohérence entre discours et acte, qui deviendra un primat de la praxis, puis une propagande par l'action, est partagée. «Nous avons appris que les discours sans action sont injustes ${ }^{13}$ », déclare ainsi Gudrun Ensslin. Et Helmut Gollwitzer, professeur de théologie évangélique à l'Université libre de Berlin - qui connaît les parents d'Ensslin, assimile la criminalité hitlérienne et la politique vietnamienne de Nixon, et prononcera un discours à l'enterrement d'Ulrike Meinhof, à Berlin, le 15 mai 1976 - écrit: «Ce ne sont pas de nouveaux mots, mais seulement de nouvelles actions qui donnent à la prédication chrétienne une nouvelle pertinence ${ }^{14}$.» Non une théologie du sacrement ou de la parole seule, mais une exhortation à l'action qui, seule, pour ce type de protestantisme, augmente la gloire de Dieu.

«Le compositeur n’a rien à dire, mais quelque chose à faire ${ }^{15}$ », semble leur répondre, comme en écho, Lachenmann, qui répète volontiers la formule, en la modifiant à peine dans ses écrits. La notation de sa «musique concrète instrumentale» l'atteste, qui prescrit non pas l'idéalité d'un son à atteindre et à interpréter, mais l'action de l'instrumentiste sur son instrument. C'est par ce qu'il fait que l'art est politique: l'œuvre n'exprime pas ce qu'elle met en musique - titres, textes ou livrets, slogans ou programmes, intention d'un musicien s'érigeant en apôtre ou prophète, sinon idéologie, cette sécularisation de l'eschatologie chrétienne-, mais est en soi expression. Lachenmann
9. Ibid., p. 78-79.

10. Sur l'éthique protestante et la RAF, voir Herrman, 2006, p. 644-656.

11. Cité dans Krebs, 1988, p. 38. Voir aussi Ensslin (dir.), 1964: ce recueil de textes et de poèmes a été préfacé par le compagnon d'alors d'Ensslin, Bernward Vesper. Sur le lien entre éthique chrétienne et lutte contre la bombe atomique, voir Gollwitzer, 1958[1957].

12. Voir Herrman, 2006, p. 648 sq.

13. Cité dans Aust, 1998, p. 75.

14. Gollwitzer, 1967, p. 50.

15. Lachenmann, 2009 ("Questions réponses" [1988]), p. 148. 
16. Voir Lachenmann, 1996 ("Über Luigi Nono" [1973]), p. 259-26o.

17. Heathcote, 2010, p. 341.

18. Voir, bien sûr, Weber, 2000, qui écrit, p. 251: "[L'ascèse chrétienne] entreprenait d'imposer sa méthode à la vie quotidienne temporelle, de la transformer en une vie rationnelle menée en ce monde mais qui n'était pas de ce monde ni conçue pour ce monde." Sur le lien entre éthique protestante et capitalisme, voir aussi les thèses d'Eberhard Gothein ou de s'oppose dès lors aux positions marxistes de l'époque, y compris celle de Luigi Nono, si l'on réduit l'essence de son engagement à une profession de foi en le communisme, forme idéale, voire religieuse, d'existence ${ }^{16}$. Et il lit Nono presque à ses dépens, comme celui qui, face à l'histoire et à une situation sociale, élimine les connotations dont les conventions ont chargé le matériau, et comme celui qui régénère ainsi l'écoute. L’objet immédiat de la musique n'est pas la guerre du Vietnam, le capitalisme, le cours tragique du monde, ses injustices, ses défaillances, un appel à sa transformation, sinon à la compassion, voire aux «lendemains qui chantent », mais cette écoute. Là vibrent sans doute, par le strict maintien du politique dans la sphère musicale, autrement dit dans le champ d'action du compositeur (ce qui, par ailleurs, consacre une certaine division du travail), un écho de l'éthique protestante du métier et une exhortation au Beruf, au sens de profession, mais aussi de vocation Luther ayant traduit par l'allemand Beruf le grec klèsis de saint Paul, cette action d'appeler à soi, d'invoquer, d'inviter, de convier. C'est pourquoi, Lachenmann y insiste, son art n'est pas de plaisanterie ou de provocation, ne vise pas à choquer, mais engendre quelque chose de sérieux.

Et jamais Lachenmann ne déroge à l'idée selon laquelle la musique engagée, y compris quand elle se revendique du marxisme-léninisme, ne serait qu'une «enclave dans le monde du divertissement occidental ${ }^{17}{ }^{\text {», dont }}$ les compositions, banals objets d'entertainment, voudraient détruire le système capitaliste, mais ne l'égratignent en rien. S'agit-il pour autant d'une déclinaison de la condamnation protestante des vains bavardages et de la jouissance naïve de l'existence et des plaisirs, sinon du luxe, à la faveur d'une ascèse intramondaine qui n'impose pas la mortification, restreint autant la consommation que l'incendie d'Ensslin, et libère l'enrichissement, comme le démontre Max Weber, des entraves de l'éthique traditionnelle? Nous ne saurions le dire. Mais au regard de ce qui précède, et de manière latente, Lachenmann paraît admettre la complémentarité, protestante, entre le métier comme authentique champ d'action de l'éthique et l'ascèse comme méthode radicale, conduite de vie organisée et contrôlée, sérieusement et systématiquement réglementée dans les sphères domestiques et publiques, en somme rationalisée - une rationalisation du religieux, mais aussi des systèmes économiques, des positions sociales, des attitudes politiques, des choix scientifiques et, pour ce qui nous concerne, des pratiques artistiques ${ }^{18}$.

Au sein des groupes allemands engagés dans la lutte armée, l'éthique protestante s'est entre-temps nourrie d'utopies révolutionnaires, de stratégies de guérilla sud-américaine et maoïste, mais aussi d'un absolu d'où tout intermédiaire est exclu, d'une rupture existentielle avec la vie menée avant la 
clandestinité, d'une conscience élitaire monopolisant la vérité, d'une volonté narcissique de puissance et d'une pensée apocalyptique atteignant un système autoritaire d'organisation et de direction, où une pression s'exerce sur les membres du groupe.

\section{II}

Le contexte biographique, néanmoins, ne suffit pas. Des conditions de possibilité culturelles, historiques et politiques étaient requises pour que Lachenmann pût le rendre public et l'inscrire dans une ouvre d'ampleur. Car rares furent, au moment des faits et de leurs points culminants - la campagne d'attentats de mai 1972 et l'Automne allemand de 1977 - les artistes qui osèrent évoquer la situation, à l'exception du plasticien Joseph Beuys et de cinéastes, parmi lesquels se compte notamment Rainer Werner Fassbinder. La Petite Fille aux allumettes participe donc d'un processus d'historicisation et d'esthétisation de la RAF, alors même que ses actions s'espacent et que se dessine sa prochaine dissolution. Dès 1992, celle-ci entérine l'abandon des assassinats - la dernière action sera un attentat du Commando Katharina-Hammerschmidt contre la centrale pénitentiaire de Weiterstadt le 27 mars 1993. Le 20 avril 1998, un dernier communiqué, sans titre, dit Auflösungserklärung («Déclaration de dissolution»), écrira : «Il y a presque vingt-huit ans, le 14 mai 1970, la RAF naissait dans une action de libération: aujourd'hui nous en terminons avec ce projet. La guérilla urbaine, sous la forme que lui a donnée la RAF, fait désormais partie de l'histoire ${ }^{19}$.»

Critiquant une stratégie réduite à la lutte armée, au détriment des fronts culturels et politiques, et contestant que quiconque puisse avoir droit de vie et de mort sur des êtres humains en raison de leur fonction dans le système, ce communiqué ne contiendra que quelques lignes sur les débuts du groupe et ses luttes «en solidarité avec les mouvements de libération». Mais il insistera sur l'Automne allemand et sur l'enlèvement et l'assassinat du président du patronat allemand, Hanns-Martin Schleyer, et reviendra sur la notion de «front anti-impérialiste » théorisée dans les années 1980, reconnaissant, après la chute du mur de Berlin, que le socialisme réel n’avait pas «réellement» abouti à une libération.

Les errances de la deuxième génération de la RAF et des suivantes avaient peu à peu contribué à l'implacable mise à l'index de l'«ennemi intérieur » et à l'état d'exception - aux tendances les plus autoritaires de l'État. Ce qu'espérait, en 1977 déjà, Liselotte Eder déclarant à son fils, Rainer Werner Fassbinder, dans le film L'Allemagne en automne (1977): «Le mieux, ce serait une sorte de dirigeant autoritaire qui serait tout à fait bon et gentil, qui serait
19. http://www.terrorisme.net/doc/ gauche/002_raf_auf.htm (consulté le 19 janvier 2018) (traduction modifiée). 
quelqu'un de bien ${ }^{20}$.» Cependant, en visant non des individus, mais les représentants de fonctions politiques, économiques, policières, juridiques, militaires..., la RAF n'avait pas œuvré à un terrorisme de masse, aveugle comme celui du massacre de l'aéroport de Lod par l'Armée rouge japonaise, le 30 mai 1972, ou celui, djihadiste, que nous connaissons aujourd'hui. Cette «rationalisation» de la terreur renforce, certes, les tentations conservatrices, mais les encadre aussi, permettant à la gauche institutionnelle ouest-allemande de légitimer et de parachever son intégration à la légalité démocratique libérale et de consolider sa social-démocratisation, qui était à l'œuvre dès les années 1960.

Contrairement à l'Espagne d'Euskadi Ta Askatasuna, à l'Italie des Brigate rosse, au Japon de la Nihon Sekigun, pour lesquels la production artistique reste limitée, la Rote Armee Fraktion suscite, depuis la fin des années 1980, une prolifération d'œuvres poétiques, romanesques, dramaturgiques, plastiques, cinématographiques... Sa fin n'est pas la seule raison. Citons l'aptitude accrue de l'art à représenter la destruction et à assimiler sa négation, comme les mutations sociologiques, la marginalisation des utopies marxistes, la dépolitisation du politique par la culture et le marché, l'élargissement des contrôles exercés par les États et l'économie mondialisée, les nouveaux équilibres stratégiques (avec l'écroulement du bloc de l'Est où se réfugièrent plusieurs militants), l'évolution des seuils de violence que tolèrent les sociétés occidentales, l'instauration de l'état d'exception, désormais structurel, la suspension théologique de l'histoire qui s'y rapporte... Et, parmi les créateurs: Franz Ackermann, Bettina Allamoda, Patrick Beurard-Valdoye, Dara Birnbaum, Don DeLillo, Friedrich Christian Delius, Michel Deutsch, HansPeter Feldmann, Rainald Goetz, Reinhard Hauff, Christopher Hein, Jörg Immendorff, Elfriede Jelinek, Martin Kippenberger, Bruce LaBruce, Claude Lévêque, Dea Loher, Heiner Müller, Jean-Gabriel Périot, Sigmar Polke, Thomas Ruff, Helma Sanders-Brahms... La liste est loin d'être exhaustive, qui ne cesse de s'allonger en Allemagne et ailleurs, notamment aux ÉtatsUnis, dans le sillage de Gerhard Richter, dont le cycle 18 octobre 1977 (1988) a été acquis par le Museum of Modern Art de New York en 1995 21. Et en 2005, après bien des débats, l'exposition «Zur Vorstellung des Terrors: die RAFAusstellung» («De la représentation de la terreur: l'exposition RAF»), dont l'un des commissaires sera le fils d'Ensslin, se tiendra au KW Institut for Contemporary Art de Berlin.

La Petite Fille aux allumettes se situe avant cette exposition, mais après le cycle de Richter à partir de photographies des hebdomadaires Stern et Der 
la musique écrite, sinon savante, occidentale, Lachenmann est assez seul à se confronter à cet épisode historique ${ }^{22}$. L'avait précédé Luigi Nono, qui avait envisagé une œuvre, inaboutie, Stammheim - Non un mistero - Infinito (Stammheim - Pas un mystère - Infini) ou Post-Prce-Ludium Stammheim (Post-Pré-Lude Stammheim), et avait expérimenté au Studio de la Fondation Heinrich-Strobel (Fribourg-en-Brisgau) des sons d'une extraordinaire violence, écrivant à même le corps de chanteurs, leur gorge, la trace d'un étranglement qui avait conduit Meinhof au suicide par pendaison, le 9 mai 1976. Sa source: un livre de Pieter Bakker Schutt, Stammheim, que Lachenmann lui avait offert en mars $1987^{23}$ et dans lequel est cité un texte de Meinhof datant de sa mise à l'isolement (1972-1973) dans un quartier insonorisé de la prison de Cologne-Ossendorf. Meinhof y décrit le sentiment d'être sans voix, d'avoir à hurler pour être audible, le chuchotement comme un cri, le supplice des sifflantes, la perte de l'équilibre..., témoignant des conséquences de la privation sensorielle qu'elle subissait. La réalité acoustique de sa camera silens, le son comme torture et cette conscience extrême du silence, semblable à des électrochocs, auraient-ils eu des incidences sur l'œuvre, d'autant que Nono recentrait alors la vocalité sur la dure consonne? «Sich selbst hören», écrit l'une des esquisses de Post-Prce-Ludium Stammheim. "S'écouter soi-même. »

Qu'en est-il, dans La Petite Fille aux allumettes, du lien entre musique, politique et éthique? Autrement dit, comment définir l'éthique compositionnelle de Lachenmann?

1. C'est un refus de la norme. Contre une convention qui reproduit, docilement, les valeurs de la classe dominante - et dont cette dernière use comme moyen de refoulement -, contre un langage contribuant à une immobilité sociale, et qu'il convient de transgresser, de surmonter, de «relever ", au double sens de aufheben, l'œuvre retire ce qui voile l'écoute. Trois points en découlent: a) Si l'œuvre évite, écarte, dissout moralement les sédimentations idéologiques, le faire artistique ne peut se dire seulement productif, mais suppose une praxis qui s'exerce à la censure et au non-être. Éthique, politique, voire religieux, Lachenmann s'oppose de la sorte au sophisme d'un poièsis comme machination, qui mène à bien, à terme, cette forme capitaliste de production dont Marx a démontré l'essence servile. b) Naît une nouvelle conception du beau, qui ne tient ni de la beauté classique, par nature mimétique, ni du sublime, à la suite de Kant et de ses avatars contemporains, mais d'un beau critique et agissant, qui se fait en écartant, et vice versa, et d'où n'est en rien exclue une dimension cathartique (ce qu'atteste la notion de «pureté»). c) L'éthique lachenmanienne suppose par conséquent qu'il n’y a pas d'écoute en soi, mais des modalités d'écoute, des écoutes en acte, reflets
22. Voir aussi, récemment, $L a$ Philosophie dans le boudoir (2015-2016), théâtre musical de François Sahran, dont une section porte sur l'usage étatique du cerveau d'Ulrike Meinhof.

23. Sur les relations entre Nono et Lachenmann, voir Nonnenmann, 2013. 
d'une pratique sociale, des forces qui la traversent et des conditions qu'elle véhicule.

2. Le toucher, chez Lachenmann, le contact, comme co-tact, de soi et de l'autre, ouvre une seconde voie, celle de la biopolitique, par laquelle Michel Foucault désignait la manière dont le pouvoir tend à se transformer, à la fin du XVIII ${ }^{\mathrm{e}}$ siècle, afin de gouverner les individus au moyen d'un certain nombre de dispositifs, de stratégies et de procédés disciplinaires, mais aussi ce que l'État constituait en population par la gestion de la vie, sinon de l'espèce ou de la race, et donc de la santé, de l'hygiène, de l'alimentation et de la natalité, en vue de produire et d'ordonner des forces, et d'accroître leur rationalité et leur utilité. Si, par les grèves de la faim, par la mort - celle des leurs et celle de leurs victimes -, par une thanatopolitique, les mouvements terroristes, en Allemagne comme ailleurs, retirent à l'État cet exercice du pouvoir sur leur corps propre et sur les corps de l'État (justice, police, armée...) dont ils exécutent des représentants, le champ ouvert par le toucher de Lachenmann se formulerait ainsi : le pouvoir œuvrant, par une anatomopolitique et une biopolitique, à surveiller et redresser les populations - y compris dans les salles de concert, les orchestres symphoniques et les théâtres lyriques, voire les modes de jeu séculaires, le corps instrumental comme produit d'une série de forces et de tensions, d'énergies, modifié par l'inexorable cycle d'actions et de réactions, de touchers et de résistances - est-il le lieu d'émergence d'un contre-pouvoir?

Or, ce qui frappe dans la lettre de Gudrun Ensslin citée dans La Petite Fille aux allumettes, c'est son énigmatique conclusion: «Écrivez sur notre peau. » Lachenmann a saisi que cette peau n'implique pas seulement le sens du toucher, mais aussi les sensations de pression (dans le traitement instrumental), de douleur (par les résonances existentielles de la lettre) et de chaleur (le saisissement, l'ascension hivernale, la marche épuisante dans la neige, la plongée dans le froid qui emportera la petite fille d'Andersen). C'est donc, en l'œuvre, une mesure tactile, mais aussi énergique, algique et thermique de cette lettre écrite en 1973, lors de la première grève de la faim des détenus de la RAF, du 17 janvier au 12 février, pour protester contre leurs conditions de détention. Le numéro 15b («Écrivez sur notre peau») est une cadenza parlando: les deux timbales et les cinq percussionnistes dessinent des mouvements, notés par des flèches, sur la peau de leurs instruments aucune métaphore, donc, dans le traitement du texte de Gudrun Ensslin, pris compositionnellement à la lettre. Dans une nuance fortissimo, des doubles croches ou des groupes de deux à huit doubles croches (exceptionnellement jusqu'à douze, mesure 264) laissent bientôt place à une improvisation sur les mêmes gestes, limitée à cinq doubles croches. Ce qui importe dans cette 
écriture des peaux, c'est la longueur, en doubles croches, des groupes, les écarts de durée entre ces groupes et l'épaisseur instrumentale sur chacune des doubles croches. Le chœur, divisé en quatre groupes identiques (SATB), donne ensuite le texte en homorythmie (schreibt), puis déconstruit (auf unsere Hand), en une polyphonie rompant l'écriture rythmique antérieure - une rupture qu'accentue le contrepoint de la première timbale, laquelle dit déjà l'incipit du numéro 18 de la partition, le début du fragment de Léonard de Vinci: «Mais moi, je vais errant, poussé par mon désir ardent.» La seconde timbale reprend les groupes de doubles croches, mais alors troués de silences de plus en plus longs. Pour cette lettre, Lachenmann adopte en outre la forme liturgique et répétitive de la litanie, nouant musique, histoire politique et éthique religieuse. Une intercession qui évoque aussi le verbe de la RAF, lequel ne tenait pas du dialogue, mais d'un dévoiement, par ses répétitions ou ses incantations, de l'éthique dans un moralisme.

\section{BIBLIOGRAPHIE}

Aust, Stefan (1998[1985]), Der Baader Meinhof Komplex, Munich, Goldmann.

Ensslin, Gudrun (dir.)(1964), Gegen den Tod: Stimmen deutscher Schriftsteller gegen die Atombombe, Stuttgart, Studio Neue Literatur.

FASSBINDER, Rainer Werner (1987), L'Anarchie de l'imagination, Paris, L'Arche.

Feneyrou, Laurent (2017), De lave et de fer. Une jeunesse allemande: Helmut Lachenmann, Paris, Éditions MF.

Gollwitzer, Helmut (1958[1957]), Les chrétiens et les armes atomiques, Genève, Labor et Fides.

Gollwitzer, Helmut (1967), Vietnam, Israel und die Christenheit, Munich, Kaiser.

Heathсоте, Abigail (2010[2006]), «Sound Structures, Transformations, and Broken Magic: An Interview with Helmut Lachemann », in Irène Deliège et Max Paddison (dir.), Contemporary Music: Theoretical and Philosophical Perspectives, Aldershot, Ashgate, p. 331-348.

Herrman, Jörg (2006), «"Unsere Söhne und Töchter” : Protestantismus und RaF-Terrorismus in den 1970er Jahren", in Wolfgang Kraushaar (dir.), Die RAF und der linke Terrorismus, Hambourg, Hamburger Edition, vol. 1, p. 644-656.

Kaltenecker, Martin (2001), Avec Helmut Lachenmann, Paris, Van Dieren.

Krebs, Mario (1988), Ulrike Meinhof: Ein Leben im Widerspruch, Hambourg, Rowohlt.

Lachenmann, Helmut (1996[1973]), «Über Luigi Nono», in Musik als existentielle Erfahrung: Schriften 1966-1995, Wiesbaden, Breitkopf \& Härtel, p. 259-260.

Lachenmann, Helmut (2009[1988]), «Questions - réponses: entretien avec Heinz-Klaus Metzger », in Écrits et entretiens, choisis et préfacés par Martin Kaltenecker, Genève, Contrechamps, p. 143-162.

Lachenmann, Helmut (2009[1993]), «Des paradis éphémères: entretien avec Peter Szendy », in Écrits et entretiens, choisis et préfacés par Martin Kaltenecker, Genève, Contrechamps, p. 193-202.

Lachenmann, Helmut (2009[2001]), «Les sons représentent des événements naturels: entretien avec Klaus Zehelein et Hans Thomalla», in Écrits et entretiens, choisis et préfacés par Martin Kaltenecker, Genève, Contrechamps, p. 247-252. 
Nonnenmann, Rainer (2013), Der Gang durch die Klippen: Helmut Lachenmanns Begegnungen mit Luigi Nono anhand ihres Briefwechsels und anderer Quellen 1957-1990, Wiesbaden, Breitkopf \& Härtel.

Schut, Pieter Bakker (dir.)(1987), Das Info : Briefe von Gefangenen aus der RAF aus der Diskussion 1973-1977, Kiel, Neuer Malik.

STORR, Robert (2000), Gerhard Richter «October 18, 1977», New York, The Museum of Modern Art.

Weber, Max (2000[1905]), L'éthique protestante et l'esprit du capitalisme, Paris, Flammarion.

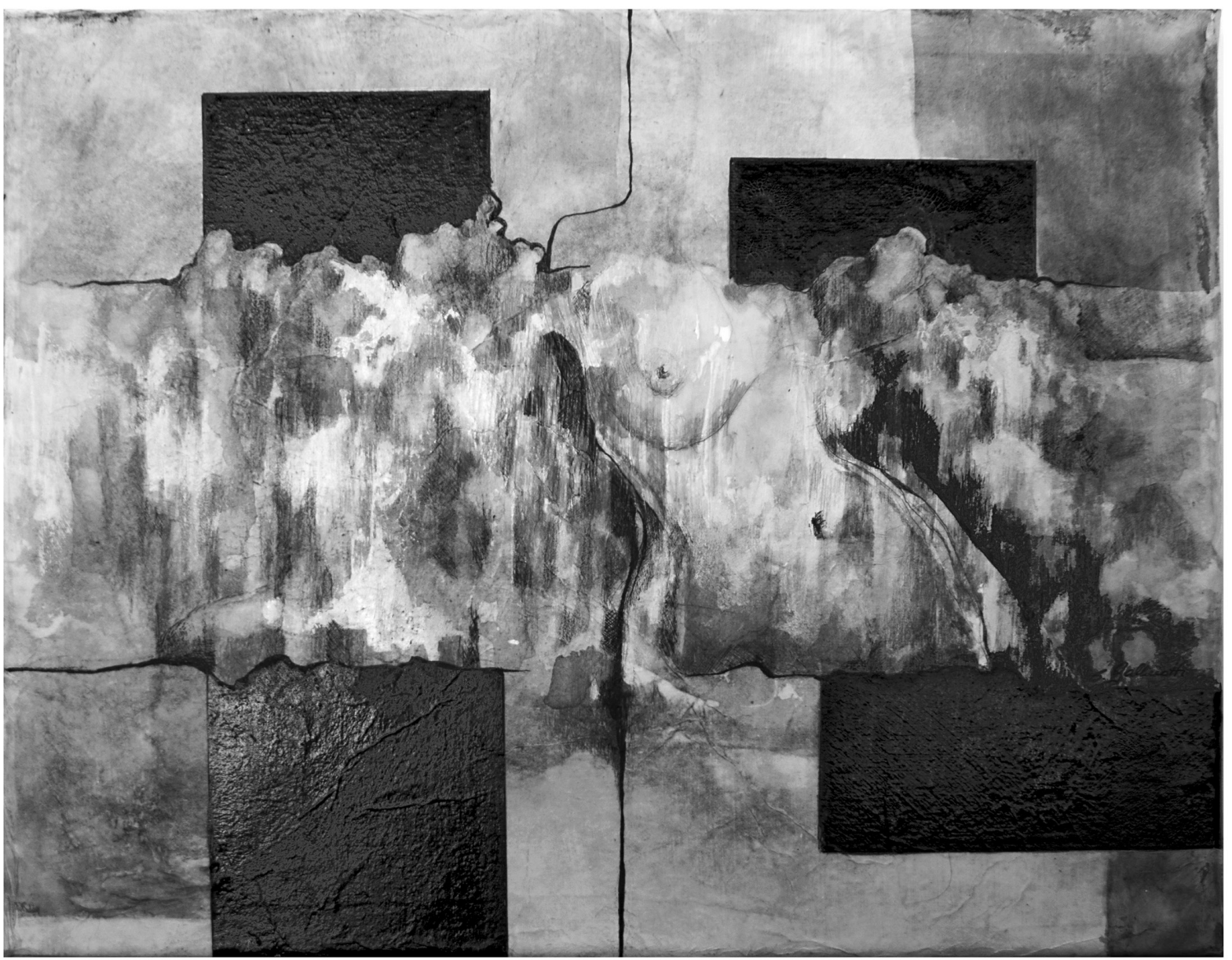

Dulce condena, 2018. Technique mixte sur toile, 32,5 × $41 \mathrm{~cm}$. Photo : Sergio Santamaría Borges 